TITLE:

\title{
Three-dimensional tomographic features of dome-shaped macula by swept-source optical coherence tomography.
}

\section{AUTHOR(S):}

Ellabban, Abdallah A; Tsujikawa, Akitaka; Matsumoto, Akiko; Yamashiro, Kenji; Oishi, Akio; Ooto, Sotaro; Nakata, Isao; ... Radwan, Tarek M; Zaky, Khaled A; Yoshimura, Nagahisa

\section{CITATION:}

Ellabban, Abdallah A ... [et al]. Three-dimensional tomographic features of dome-shaped macula by swept-source optical coherence tomography.. American journal of ophthalmology 2013, 155(2): 320-328.e2

\section{ISSUE DATE:}

2013-02

URL:

http://hdl.handle.net/2433/169953

\section{RIGHT:}

(C) 2013 Elsevier Inc.; This is not the published version. Please cite only the published version.; この論文は出版社版でありません。引用の際に は出版社版をご確認ご利用ください。 


\begin{abstract}
PURPOSE: To study the tomographic features and pathomorphology of dome-shaped maculas with swept-source optical coherence tomography (OCT).

DESIGN: Prospective cross sectional study.

METHODS: The macular area of 51 highly myopic eyes (35 patients) with dome-shaped macula was studied with swept-source OCT at 1,050 nm. Three-dimensional (3D) datasets were obtained with raster scanning covering a $12 \times 8 \mathrm{~mm}^{2}$ area; 3D images of the posterior pole were constructed by auto-segmentation of the retinal pigment epithelium (RPE).

RESULTS: In all reconstructed 3D images of the RPE, two outward concavities were seen within the posterior staphyloma and a horizontal ridge was formed between these two concavities. In 42 of these eyes, this horizontal ridge was band-shaped. The vertical OCT section through the fovea showed a convex configuration of RPE, but the horizontal section showed an almost flat RPE line. In nine eyes, 3D images showed a typical dome-shaped convexity within the staphyloma. OCT scans showed no outward protrusions in the external scleral surface, but marked scleral thinning was seen consistent with the two outward concavities of the RPE. The sclera of the fovea (518.6 \pm $97.6 \mu \mathrm{m}$ ) was significantly thicker than that in all four quadrants of the parafoveal area (277.2 - $360.3 \mu \mathrm{m}, P<.001)$.
\end{abstract}

CONCLUSIONS: In highly myopic eyes with a dome-shaped macula, a horizontal ridge is formed within the posterior staphyloma by uneven thinning of the sclera. 


\title{
THREE-DIMENSIONAL TOMOGRAPHIC FEATURES OF DOME-SHAPED MACULA BY SWEPT-SOURCE OPTICAL COHERENCE TOMOGRAPHY
}

Short title: THREE-DIMENSIONAL FEATURES OF DOME-SHAPED MACULA

\author{
ABDALLAH A. ELLABBAN, ${ }^{1,2}$ AKITAKA TSUJIKAWA, ${ }^{1}$ AKIKO MATSUMOTO, ${ }^{1,3}$ KENJI \\ YAMASHIRO, ${ }^{1}$ AKIO OISHI, ${ }^{1}$ SOTARO OOTO, ${ }^{1}$ ISAO NAKATA, ${ }^{1}$ YUMIKO \\ AKAGI-KURASHIGE, ${ }^{1}$ MASAHIRO MIYAKE, ${ }^{1}$ HUSSEIN S. ELNAHAS, ${ }^{2}$ TAREK M. \\ RADWAN, ${ }^{2}$ KHALED A. ZAKY, ${ }^{2}$ AND NAGAHISA YOSHIMURA ${ }^{1}$
}

From ${ }^{1}$ the Department of Ophthalmology and Visual Sciences, Kyoto University Graduate School of Medicine, Kyoto, Japan; ${ }^{2}$ the Department of Ophthalmology, Suez Canal University, Faculty of Medicine, Ismailia, Egypt; ${ }^{3}$ Topcon Corporation, Tokyo, Japan.

Supplemental Material available at AJO.com

Correspondence to Akitaka Tsujikawa, MD, Department of Ophthalmology and Visual Sciences, Kyoto University Graduate School of Medicine, Sakyo-ku, Kyoto 606-8507, Japan; phone: +81-75-751-3260; fax: +81-75-752-0933; e-mail:

tujikawa@kuhp.kyoto-u.ac.jp 


\section{INTRODUCTION}

High myopia is one of the leading causes of visual disturbance worldwide, and is particularly prevalent in Asians. ${ }^{1-3}$ Axial elongation of the globe leads to complex changes in the topography of the posterior pole, with concomitant thinning of the retina, choroid, and sclera, and subsequent development of macular pathologies. ${ }^{4-6}$ Posterior staphyloma, which is one of the characteristic features of high myopia, has been reported to be associated with various vision-threatening complications, including choroidal neovascularization, macular holes, schisis, chorioretinal atrophy, and visual field defects. ${ }^{4-6}$ Using fundus examination, Curtin ${ }^{4}$ classified posterior staphyloma in eyes with high myopia into 10 types.

Based on optical coherence tomography (OCT), dome-shaped macula was first described by Gaucher and associates ${ }^{7}$ as an unexpected finding in myopic staphyloma, and was characterized as an inward convexity of the macula that occurred in highly myopic eyes within the concavity of a posterior staphyloma. They suggested that dome-shaped macula might be due to changes in choroidal thickness or to changes in scleral shape in highly myopic eyes. Thereafter, Imamura and associates, ${ }^{8}$ by using enhanced depth imaging $\mathrm{OCT},{ }^{9}$ reported that dome-shaped macula is due to a localized variation in thickness of the sclera in the macular area. However, the mechanisms which underlie this unusual tomographic feature remain unknown. In addition, most cases of dome-shaped macula in these previous reports were examined by only unidirectional OCT scans, ${ }^{7,8,10,11}$ So far, little information is available on the topography of the posterior pole in eyes with dome-shaped macula.

With the advances in OCT in the last decade, several retinal pathologies have been identified in highly myopic eyes. ${ }^{12-19}$ However, imaging of highly myopic eyes with conventional OCT systems has several difficulties. Recent OCT generation has utilized swept-source laser as a light source..$^{20-27}$ The swept-source OCT at a longer wavelength allows deeper tissue penetration into the choroid and even sclera, especially in highly myopic eyes. Additionally, the higher imaging speed, larger scan window, and lower sensitivity roll-off with depth allow for larger scans and the production of three-dimensional (3D) images of the posterior pole. In the study described herein, we examined eyes with dome-shaped macula using swept-source OCT at a longer wavelength in order to study the pathomorphology of dome-shaped macula by 3D imaging and to elucidate its association with macular complications. 


\section{PATIENTS AND METHODS}

In the current prospective cross-sectional study, we examined 51 eyes (35 patients) with high myopia that showed a dome-shaped macular configuration within the posterior staphyloma. The macular area of the study subjects was examined using a prototype swept-source OCT at Kyoto University Hospital between the beginning of September 2010 and the end of February 2012. The diagnosis of dome-shaped macula was based on the OCT findings of an inward bulge inside the chorioretinal posterior concavity of the macular area, per Gaucher and associates. ${ }^{7}$ This unusual feature of the macula in eyes with dome-shaped macula is especially clear on the vertical scans, ${ }^{7}$ and performing long vertical scans has been recommended in order to discern specific features. ${ }^{28}$ In the current study, we recruited patients with highly myopic eyes with dome-shaped macula on the basis of the presence of an inward bulge of the retinal pigment epithelium (RPE) of more than $50 \mu \mathrm{m}$ on vertical sections of OCT (Figure 1) above a presumed line tangent to the outer surface of RPE at the bottom of the posterior staphyloma. High myopia was defined as a refractive error of -6.0 diopters or more and/or an axial length of $26.5 \mathrm{~mm}$ or greater. Eyes were excluded if they had a preexisting ocular disease or if they had a history of ocular surgery, other than cataract surgery. The original refractive errors were used for eyes that underwent cataract surgery. The type of posterior staphyloma was determined by location and size according to the classification of Curtin. ${ }^{4}$ In the current study, eyes with an inferior posterior staphyloma (type $\mathrm{V}$ ) were excluded. All eyes of our patients had a posterior staphyloma of type I, II, III, or IX. The Institutional Review Board and Ethics Committee of Kyoto University approved this prospective study, which adhered to the tenets of the Declaration of Helsinki. Written informed consent for research participation was obtained from each subject before examination.

All subjects underwent a comprehensive ocular examination, including autorefractometry (ARK1, Nidek, Gamagori, Japan), best-corrected visual acuity measurement with a 5-m Landolt chart, axial length measurement using ocular biometry (IOLMaster, Carl Zeiss Meditec, Jena, Germany), slit-lamp examination, intraocular pressure measurement, dilated color fundus photography (TRC50LX, Topcon Corp., Tokyo, Japan), and swept-source OCT examination. All eyes with macular complications underwent simultaneous fluorescein angiography and indocyanine green angiography using Spectralis HRA+OCT (Heidelberg Engineering, Heidelberg, Germany). Eyes with poor images due to opaque media (e.g., cataracts or corneal opacity) were excluded from the study.

\section{SWEPT-SOURCE OCT AND SCAN PROTOCOLS}


The prototype swept-source OCT (Topcon Corp.) used in the current study has been previously described. ${ }^{20,21}$ In brief, this swept-source OCT uses a light source of a wavelength-sweeping laser centered at $1050 \mathrm{~nm}$, with a repetition rate of $100,000 \mathrm{~Hz}$. This OCT system has a depth of $2.6 \mathrm{~mm}$ in the scan window. Swept-source OCT examinations of the eligible subjects were performed by trained examiners after pupil dilation.

In each subject, multi-averaged horizontal and vertical raster scans of $12 \mathrm{~mm}$ were obtained. Fifty single images, where each image consisted of 1,024 A-scans, were registered and averaged by software to create a multi-averaged single image. The vertical scan was centered on the fovea while the horizontal scan was centered on the midpoint between the fovea and optic disc.

3D imaging datasets were acquired by using a raster scan protocol of 512 (horizontal) $\times 128$ (vertical) A-scans per dataset in 0.8 seconds. Each raster scan consisted of $128 \mathrm{~B}$-scans and covered an area of $12 \times 8 \mathrm{~mm}^{2}$ centered on the fovea. Centration was confirmed by internal fixation and by a built-in camera within the swept-source OCT system. To improve signal to noise ratio, each image was de-speckled by the weighted moving averaging of three consecutive original B-scans. In each image, the RPE line was determined using the built-in automated segmentation-modifying tool, with manual corrections if necessary. 3D images were constructed to represent the curvature of the posterior pole.

\section{CHOROIDAL AND SCLERAL THICKNESS MEASUREMENT PROTOCOL}

Using the multi-averaged OCT images, we measured the choroidal and scleral thicknesses at the center of the fovea and at surrounding parafoveal regions at $2000 \mu \mathrm{m}$ superiorly, inferiorly, temporally and nasally, respectively. Retinal thickness was defined as the distance between the vitreoretinal interface and the outer border of the RPE; choroidal thickness was defined as the distance between the outer border of the RPE line and the chorioscleral interface; scleral thickness was defined as the distance between the chorioscleral interface and outer scleral border. Retinal, choroidal, and scleral thicknesses at the fovea were calculated as the average of the measurements in the vertical and horizontal OCT images at the fovea. We also measured the height of the inward bulge of the RPE above a presumed line tangent to the RPE at the bottom of the posterior staphyloma (Figure 1).

The outer surface of the sclera was carefully identified in the OCT scans from retroocular structures such as episcleral tissue, Tenon's capsule, inferior oblique aponeurosis or orbital fat. Scleral tissues were identified by their lamellar morphology, continuity of the outer surface of the sclera within the 7 raster lines in the vertical and 
horizontal scans and high reflectivity values. The outer border of the sclera at the fovea exceeded the scan window in two eyes and the outer scleral border was not clearly visible in three eyes. In these five eyes, we used the greatest measurable scleral thickness. In the parafoveal region, the scleral thickness could be identified in all eyes.

\section{STATISTICAL ANALYSIS}

Statistical analysis was computed using SPSS statistical software (version 16; SPSS Inc., Chicago, IL, USA). All values are expressed as mean \pm standard deviation. The measured visual acuity was converted to the logarithm of the minimum angle of resolution (logMAR) for statistical analysis. The data were analyzed using the unpaired t-test. The Fischer exact test was used to analyze categorical variables. Choroidal and scleral thicknesses within different regions were compared using one-way analysis of variance with Tukey post-hoc analysis. A $P$ value of less than .05 was considered to be statistically significant. 


\section{RESULTS}

In the current study, 51 eyes of 35 patients (8 men and 27 women) with dome-shaped macula were studied by swept-source OCT at $1 \mu \mathrm{m}$ wavelength. The characteristics of the subjects are summarized in Table 1. Their mean age was $65.6 \pm 11.3$ years (range: 40 to 87 years). Mean refractive error was $-13.69 \pm 5.86$ diopters (range: -6.75 to -31.00 diopters) and mean axial length was $29.53 \pm 2.16 \mathrm{~mm}$ (range: 26.16 to $34.89 \mathrm{~mm}$ ). Mean visual acuity in logMAR was $0.36 \pm 0.39$ (range: -0.18 to 1.40 ). The dome-shaped macula was bilateral in $16(45.7 \%)$ patients and all patients were Japanese.

\section{SHAPE OF INWARD PROTRUSION IN EYES WITH DOME-SHAPED MACULA}

Multi-averaged sections with the swept-source OCT enabled clear visualization of the structure of the retina, choroid, and sclera. In all eyes, an inward bulge within the posterior staphyloma was seen clearly in the vertical sections. The height of the inward bulge above the tangent plane was $152.3 \pm 58.8 \mu \mathrm{m}$ (range: 56.0 to $294.0 \mu \mathrm{m}$ ). In the horizontal scan, however, the inward convexity was often detected less clearly. In some eyes, the horizontal scan showed a flat RPE line without any inward bulge within the staphyloma (Figure 2).

Reconstructed 3D images revealed curvature of the RPE in the posterior pole. In all eyes, two outward concavities of RPE were seen within the posterior staphyloma and a horizontal ridge was present between these two concavities, which was located at the fovea (Figure 3, supplemental movie 1, supplemental Figures 1 and 2). In 42 eyes $(82.4 \%)$, the horizontal ridge was present as a band-shape and the vertical scan through the fovea showed a convex configuration, but the horizontal scan showed an almost flat RPE line. In the other nine eyes (17.6\%), 3D imaging of the RPE showed a typical dome-shaped convexity within the staphyloma (Figure 4) and both vertical and horizontal OCT scans showed a convex configuration. Of these nine eyes, the top of the convexity was located beneath the fovea in seven eyes and was most prominent at the nasal side of the fovea in two eyes.

In two patients, one eye showed the typical dome-shaped convexity and the other eye showed a band-shaped ridge. There were no significant differences in axial length, refractive error, or choroidal thickness at the fovea, or the height of the inward bulge between these two types. However, the foveal scleral thickness was significantly thicker in eyes with a dome-shaped convexity $(598.3 \pm 76.8 \mu \mathrm{m})$ than in eyes with a band-shaped ridge $(501.5 \pm 93.6 \mu \mathrm{m}, P=.006)$.

\section{THICKNESS OF THE SCLERA IN DOME-SHAPED MACULA}


Table 2 shows thickness of the choroid and of the sclera in the posterior pole. The choroid showed generalized thinning of the entire posterior pole $(34.5 \pm 29.0 \mu \mathrm{m}$ in the fovea). In OCT scans, no outward protrusions were seen in the external surface of the sclera. However, the sclera in the posterior pole showed an uneven thickness consistent with the two outward concavities of RPE within the staphyloma. The OCT sections showed marked scleral thinning. In the fovea, the sclera was relatively thick (518.6 \pm 97.6 $\mu \mathrm{m})$, compared with parafoveal areas in all four quadrants $(277.2-360.3 \mu \mathrm{m}, P<.001$, respectively). In addition, the scleral thickness was more markedly reduced in the superior and inferior parafoveal areas than in the nasal or temporal parafoveal areas. The scleral thickness at $2000 \mu \mathrm{m}$ superiorly and inferiorly was significantly less than that nasally $(P<.001$ and $=.001$, respectively).

\section{MACULAR COMPLICATIONS}

Table 3 shows the macular complications seen in our patients. Of the 51 eyes with dome-shaped macula, a full-thickness macular hole was seen in only one (1.9\%). Extrafoveal retinal schisis was seen in nine eyes (17.6\%), but foveal schisis was seen in only one (1.9\%). Twenty-one eyes (41.2\%) showed choroidal neovascularization (CNV). Of these 21eyes, 12 had been treated with anti-vascular endothelial growth factor (VEGF) agents, 4 had received photodynamic therapy and 2 had received photodynamic therapy combined with anti-VEGF agents.

Table 4 shows the comparison between eyes with CNV and eyes without CNV in the current study subjects. Visual acuity in logMAR was significantly poorer in eyes with CNV $(0.53 \pm 0.42)$ than in eyes without CNV $(0.24 \pm 0.32, P=.007)$, but there were no differences in axial length, refractive error, or retinal, choroidal, or scleral thickness at the fovea between these two groups. However, the mean height of the inward bulge was significantly less in eyes with CNV $(131.0 \pm 51.7 \mu \mathrm{m})$ than in eyes without CNV $(167.3 \pm$ $59.5 \mu \mathrm{m}, P=.028)$. 


\section{DISCUSSION}

With the use of a light source at this longer wavelength, the current swept-source OCT allows high-contrast, long-penetration imaging of the entire choroid and sclera. When eyes with high myopia are scanned with a conventional OCT, the obtained images are often partially inverted or folded due to the limit of the scan window. The tunable laser source of the swept-source OCT has a lower signal decay versus depth compared to the existing spectral-domain OCT systems. Having a long scan window depth, the swept-source OCT used in the current study allows entire line scans of $12 \mathrm{~mm}$, even in highly myopic eyes with posterior staphyloma. In addition, high-speed scanning coupled with the high sensitivity allowed high density scanning of the macular area $\left(12 \times 8 \mathrm{~mm}^{2}\right)$. By segmentation of the RPE line by means of the automated built-in software, we constructed 3D images of the curvature of the posterior pole in eyes with dome-shaped macula.

Dome-shaped macula was first described by Gaucher and associates ${ }^{7}$ in 2008 as an inward bulge of the macula in highly myopic eyes within the concavity of posterior staphyloma. However, there is no precise definition of dome-shaped macula. Originally, the authors reported that this unusual feature of the macular profile is especially clear on vertical scans, ${ }^{7}$ and Coco and associate ${ }^{28}$ recommended performing long vertical scans to identify this feature. Based on these previous reports, we used numerical data for the presence of an inward bulge of the RPE of more than $50 \mu \mathrm{m}$ on the vertical OCT section above a presumed line tangent to the RPE at the bottom of the posterior staphyloma. On vertical scans, the height of the inward bulge ranged from $56.0 \mu \mathrm{m}$ to $294.0 \mu \mathrm{m}$. On horizontal scans, however, the inward convexity was often detected less clearly and only nine eyes showed an inward bulge of more than $50 \mu \mathrm{m}$. A recent report by Coco and associates ${ }^{28}$ supports our current findings; when eyes with dome-shaped macula were examined with OCT, an inward bulge of the macula was detected on both vertical and horizontal scans in nine eyes, but only on the vertical scan in 26 eyes.

In reconstructed 3D images of the RPE, two outward concavities were seen within the posterior staphyloma and a horizontal ridge was formed between these two concavities in all eyes. Unexpectedly, this horizontal ridge was present in the typical dome-shaped convexity in only nine of our 51 eyes; the remaining 42 eyes showed a band-shaped ridge that extended horizontally from the optic disc through the fovea. However, statistical analysis showed no major difference between the two types of protrusion. In addition, two of our patients showed a band-shaped ridge in one eye and a dome shaped convexity in the other eye. A dome-shape convexity that is generally recognized as dome-shaped macula may be a variant of the more common band-shaped 
ridge between a pair of outward concavities within the posterior staphyloma.

Recently, Moriyama and associate ${ }^{29}$ studied the topography of the highly myopic eyes with high resolution MRI. Although 3D MRI reconstructs the shape of the vitreous cavity, it does contribute to our understanding of the entire shape of eyeballs with high myopia. Those authors reported that $63.3 \%$ of eyes with high myopia had more than one protrusion on the vitreous cavity and that eyes with three protrusions showed dome-shaped macula appearance on OCT sections. These eyes had one nasal protrusion and another temporal protrusion that was divided into two parts, superior and inferior, separated by the macula. Their observation supports our current finding of two outward concavities of the RPE within the posterior staphyloma. However, we could not confirm the presence of the nasal concavity in our patients because it is sometimes beyond the limit of our scan. In nine eyes with dome-shaped convexity, the top of the convexity was located under the fovea in seven eyes and was most prominent at the nasal side of the fovea in two eyes. The third outward concavity on the nasal side might contribute to formation of the dome-shaped convexity on the horizontal ridge.

In our patients, no outward protrusions were seen in the external surface of the sclera, although two outward concavities of RPE were seen within the posterior staphyloma. Imamura and associates ${ }^{8}$ suggested that dome-shaped macula is the result of a relative localized variations in thickness of the sclera beneath the macula. Consistent with their report, the foveal scleral thickness in our patients was significantly greater than that of the parafoveal regions in all four quadrants. In addition, OCT sections showed marked scleral thinning, consistent with two outward concavities within the staphyloma. In our patients, scleral thickness at the fovea $(518.6 \pm 97.6 \mu \mathrm{m})$ was in between data obtained from enucleated normal eyes $(0.94 \pm 0.18 \mathrm{~mm})^{30}$ and OCT measurements of highly myopic eyes $(281 \pm 85 \mu \mathrm{m}){ }^{8}$ However, the scleral thickness within the two outer concavities within the staphyloma $(277.2-284.9 \mu \mathrm{m})$ was similar to that of highly myopic eyes.

Curtin ${ }^{4}$ classified posterior staphylomas in eyes with high myopia into 10 types. Types I and II staphylomas are reported to be most common in the Japanese population, with type I being seen in $23.4 \%$ and type II in $52.7 \%$ of individuals with high myopia. ${ }^{31}$ In younger patients with high myopia, posterior staphyloma is rarely detected. The development of posterior staphyloma at an older age is often accompanied by scleral thinning. In eyes with high myopia, the location of the ectatic change can vary between individuals, ${ }^{4}$ and the posterior staphyloma has been shown to not only deepen with age but also to change its shape over time. ${ }^{31}$ So we postulate that such asymmetric expansion of the eyeball could lead to formation of the dome-shaped macular configuration within the staphyloma. This asymmetric expansion may be secondary to a 
difference in structural strength of the sclera at the fovea due to either a regional difference in the biochemical structure of scleral lamellae or to organization of the collagen bundles that make the central part of the sclera in eyes with dome-shaped macula mechanically stronger than are the upper or lower regions.

Although the mechanism of myopic CNV is still controversial, several factors have been reported to pose a risk for development of CNV, such as lacquer cracks, ${ }^{32,33}$ patchy atrophy,${ }^{34}$ choroidal thinning, ${ }^{35}$ dysfunction of choroidal circulation, ${ }^{36,37}$ and posterior staphyloma protrusion. ${ }^{35}$ In the current study, CNV was present in $41.2 \%$ of eyes, and the height of the inward bulge in eyes without CNV was significantly greater than in eyes with $\mathrm{CNV}$, although the reason for this finding is uncertain. In eyes with greater dome-shaped macula, however, the change in the foveal contour from concave to convex within the incurvation of the posterior staphyloma may ameliorate the overall protrusion of the staphyloma or may alter the mechanical damage induced by lacquer cracks, resulting in a decreased risk of CNV.

It is interesting to note that, although extrafoveal retinal schisis was present in nine eyes, only one eye had foveal schisis without either foveal detachment or macular hole formation. Although, in the population of the current study the mean spherical equivalent and axial length were relatively high, the incidence of foveoschisis was lower than in previous reports. ${ }^{15,38}$ The bulge in eyes with dome-shaped macula may act as a macular buckle-like mechanism, indenting the fovea similar to a macular exoplant or Ando plomb device, ${ }^{39-41}$ and thus prevent or alleviate tractional forces over the fovea, thereby preventing schisis or detachment.

The current study had some limitations. All measurements of choroidal and scleral thicknesses were done manually using a built-in caliper. In addition, all study subjects were Japanese. As the distribution of type of posterior staphyloma in Japanese is different from that in Caucasians, ${ }^{31}$ further studies in other ethnic groups are necessary. Furthermore, the current study is cross-sectional, so additional longitudinal studies are needed to elucidate clearly the scleral changes in the formation of dome-shaped macular configuration in eyes with high myopia. Very recently, Coco and associates ${ }^{28}$ postulated the concept of 'macular bending' defined as a smooth macular elevation found in OCT in patients with high myopia related to either dome-shaped macula or the border of inferior staphyloma. In the current study, eyes with inferior staphyloma (type V) were excluded. Although we believe that these eyes are different from those with typical dome-shaped macula, other authors have suggested that these may be variants. ${ }^{10,28}$

Gaucher and associates ${ }^{7}$ reported that all eyes with dome-shaped macula have a posterior staphyloma of type I or II, and Imamura and associates ${ }^{8}$ suggested that dome-shaped macula is a novel posterior anatomic characteristic of some highly myopic 
eyes, and is not a specific subtype of posterior staphyloma. In the current study, eyes with dome-shaped macula had either posterior staphyloma type I, II, or, less frequently, III or IX, while type $\mathrm{V}$ was excluded. We believe that dome-shaped macula is not related to the type of staphyloma but, rather, is related to an anatomic or structural change within the sclera, and can occur in eyes with any type of staphyloma. 


\section{ACKNOWLEDGMENTS}

A. Funding/Support: This study was supported in part by the Japan Society for the Promotion of Science (JSPS), Tokyo, Japan (Grant-in-Aid for Scientific Research, no. 21592256), and by the Japan National Society for the Prevention of Blindness, Tokyo, Japan.

B. Financial Disclosure: A. Matsumoto, Topcon Corporation (Employment); N. Yoshimura, Topcon Corporation (Financial Support), Nidek (Financial Support, Consultant), Canon (Financial Support).

C. Contributions of Authors: Conception and design of the study (AAE, AT, AM, HSE, TMR, KAZ, NY); analysis and interpretation (AAE, AT, AM, KY, AO, SO, IN, YA-K, MM, HSE, TMR, KAZ); writing of the article (AAE, AT); critical revision of the article (AAE, AT, AM, KY, AO, SO, IN, YA-K, MM, HSE, TMR, KAZ, NY); final approval of the article (AAE, AT, AM, KY, AO, SO, IN, YA-K, MM, HSE TMR, KAZ, NY); data collection (AAE, AM, KY, $A O, S O, I N, Y A-K, M M)$.

E. Other Acknowledgments: none. 


\section{REFERENCES}

1. Iwase A, Araie M, Tomidokoro A, Yamamoto T, Shimizu H, Kitazawa Y. Prevalence and causes of low vision and blindness in a Japanese adult population: the Tajimi Study. Ophthalmology 2006;113(8):1354-1362.

2. Xu L, Wang Y, Li Y, Cui T, Li J, Jonas JB. Causes of blindness and visual impairment in urban and rural areas in Beijing: the Beijing Eye Study. Ophthalmology 2006;113(7):1134-1141.

3. Sawada A, Tomidokoro A, Araie M, Iwase A, Yamamoto T. Refractive errors in an elderly Japanese population: the Tajimi study. Ophthalmology 2008;115(2):363-370.

4. Curtin BJ. The posterior staphyloma of pathologic myopia. Trans Am Ophthalmol Soc 1977;75:67-86.

5. Hayashi K, Ohno-Matsui K, Shimada N, et al. Long-term pattern of progression of myopic maculopathy: a natural history study. Ophthalmology 2010;117(8):1595-1611.

6. McBrien NA, Gentle A. Role of the sclera in the development and pathological complications of myopia. Prog Retin Eye Res 2003;22(3):307-338.

7. Gaucher D, Erginay A, Lecleire-Collet A, et al. Dome-shaped macula in eyes with myopic posterior staphyloma. Am J Ophthalmol 2008;145(5):909-914.

8. Imamura Y, lida T, Maruko I, Zweifel SA, Spaide RF. Enhanced depth imaging optical coherence tomography of the sclera in dome-shaped macula. Am J Ophthalmol 2011;151(2):297-302.

9. Spaide RF, Koizumi H, Pozzoni MC. Enhanced depth imaging spectral-domain optical coherence tomography. Am J Ophthalmol 2008;146(4):496-500.

10. Byeon SH, Chu YK. Dome-shaped macula. Am J Ophthalmol 2011;151(6):1101; author reply 1101-1102.

11. Mehdizadeh M, Nowroozzadeh MH. Dome-shaped macula in eyes with myopic posterior staphyloma. Am J Ophthalmol 2008;146(3):478; author reply 478-479.

12. Sayanagi K, Morimoto Y, Ikuno Y, Tano Y. Spectral-domain optical coherence tomographic findings in myopic foveoschisis. Retina 2010;30(4):623-628.

13. Costa RA, Skaf M, Melo LA Jr, et al. Retinal assessment using optical coherence tomography. Prog Retin Eye Res 2006;25(3):325-353.

14. Sayanagi K, Ikuno Y, Gomi F, Tano Y. Retinal vascular microfolds in highly myopic eyes. Am J Ophthalmol 2005;139(4):658-663.

15. Takano M, Kishi S. Foveal retinoschisis and retinal detachment in severely myopic eyes with posterior staphyloma. Am J Ophthalmol 1999;128(4):472-476.

16. Sayanagi K, Ikuno $\mathrm{Y}$, Tano $\mathrm{Y}$. Tractional internal limiting membrane detachment in highly myopic eyes. Am J Ophthalmol 2006;142(5):850-852. 
17. Shimada N, Ohno-Matsui K, Nishimuta A, et al. Detection of paravascular lamellar holes and other paravascular abnormalities by optical coherence tomography in eyes with high myopia. Ophthalmology 2008;115(4):708-717.

18. Fujiwara T, Imamura Y, Margolis R, Slakter JS, Spaide RF. Enhanced depth imaging optical coherence tomography of the choroid in highly myopic eyes. Am J Ophthalmol 2009;148(3):445-450.

19. Forte R, Cennamo G, Pascotto F, de Crecchio G. En face optical coherence tomography of the posterior pole in high myopia. Am J Ophthalmol 2008;145(2):281-288.

20. Ellabban AA, Tsujikawa A, Matsumoto A, et al. Macular choroidal thickness and volume in eyes with angioid streaks measured by swept source optical coherence tomography. Am J Ophthalmol 2012;153(6):1133-1143.

21. Hirata M, Tsujikawa A, Matsumoto A, et al. Macular choroidal thickness and volume in normal subjects measured by swept-source optical coherence tomography. Invest Ophthalmol Vis Sci 2011;52(8):4971-4978.

22. Ikuno Y, Maruko I, Yasuno Y, et al. Reproducibility of retinal and choroidal thickness measurements in enhanced depth imaging and high-penetration optical coherence tomography. Invest Ophthalmol Vis Sci 2011;52(8):5536-5540.

23. Ohno-Matsui K, Akiba M, Moriyama M, Ishibashi T, Tokoro T, Spaide RF. Imaging retrobulbar subarachnoid space around optic nerve by swept-source optical coherence tomography in eyes with pathologic myopia. Invest Ophthalmol Vis Sci 2011;52(13):9644-9650.

24. Ohno-Matsui K, Akiba M, Moriyama M, et al. Acquired optic nerve and peripapillary pits in pathologic myopia. Ophthalmology 2012;119(8):1685-1692.

25. Jirarattanasopa $P$, Ooto $S$, Tsujikawa A, et al. Assessment of macular choroidal thickness by optical coherence tomography and angiographic changes in central serous chorioretinopathy. Ophthalmology 2012;119(8):1666-1678.

26. Motaghiannezam R, Schwartz DM, Fraser SE. In vivo human choroidal vascular pattern visualization using high-speed swept-source optical coherence tomography at $1060 \mathrm{~nm}$. Invest Ophthalmol Vis Sci 2012;53(4):2337-2346.

27. Maruko I, lida T, Sugano Y, Oyamada H, Sekiryu T. Morphologic choroidal and scleral changes at the macula in tilted disc syndrome with staphyloma using optical coherence tomography. Invest Ophthalmol Vis Sci 2011;52(12):8763-8768.

28. Coco RM, Sanabria MR, Alegria J. Pathology associated with optical coherence tomography macular bending due to either dome-shaped macula or inferior staphyloma in myopic patients. Ophthalmologica 2012;228:7-12.

29. Moriyama M, Ohno-Matsui K, Hayashi K, et al. Topographic analyses of shape of 
eyes with pathologic myopia by high-resolution three-dimensional magnetic resonance imaging. Ophthalmology 2011;118(8):1626-1637.

30. Vurgese S, Panda-Jonas S, Jonas JB. Scleral thickness in human eyes. PLoS One 2012;7(1):e29692.

31. Hsiang HW, Ohno-Matsui K, Shimada N, et al. Clinical characteristics of posterior staphyloma in eyes with pathologic myopia. Am J Ophthalmol 2008;146(1):102-110.

32. Avila MP, Weiter JJ, Jalkh AE, Trempe CL, Pruett RC, Schepens CL. Natural history of choroidal neovascularization in degenerative myopia. Ophthalmology 1984;91(12):1573-1581.

33. Ikuno $Y$, Sayanagi $K$, Soga $K$, et al. Lacquer crack formation and choroidal neovascularization in pathologic myopia. Retina 2008;28(8):1124-1131.

34. Ohno-Matsui K, Yoshida T, Futagami S, et al. Patchy atrophy and lacquer cracks predispose to the development of choroidal neovascularisation in pathological myopia. Br J Ophthalmol 2003;87(5):570-573.

35. Ikuno Y, Jo Y, Hamasaki T, Tano Y. Ocular risk factors for choroidal neovascularization in pathologic myopia. Invest Ophthalmol Vis Sci 2010;51(7):3721-3725.

36. Dimitrova G, Tamaki Y, Kato S, Nagahara M. Retrobulbar circulation in myopic patients with or without myopic choroidal neovascularisation. Br J Ophthalmol 2002;86(7):771-773.

37. Wakabayashi T, Ikuno Y. Choroidal filling delay in choroidal neovascularisation due to pathological myopia. Br J Ophthalmol 2010;94(5):611-615.

38. Baba T, Ohno-Matsui K, Futagami S, et al. Prevalence and characteristics of foveal retinal detachment without macular hole in high myopia. Am J Ophthalmol 2003;135(3):338-342.

39. Theodossiadis GP, Theodossiadis PG. The macular buckling procedure in the treatment of retinal detachment in highly myopic eyes with macular hole and posterior staphyloma: mean follow-up of 15 years. Retina 2005;25(3):285-289.

40. Mateo C, Burés-Jelstrup A, Navarro R, Corcóstegui B. Macular buckling for eyes with myopic foveoschisi secondary to posterior staphyloma. Retina 2012;32(6):1121-1128.

41. Tanaka T, Ando F, Usui M. Episcleral macular buckling by semirigid shaped-rod exoplant for recurrent retinal detachment with macular hole in highly myopic eyes. Retina 2005;25(2):147-151. 


\section{FIGURE CAPTIONS}

Figure 1. Parameters associated with the dome-shaped macular configuration as measured from a multi-averaged vertical section obtained with swept-source optical coherence tomography. At the fovea, three parameters were measured: retinal thickness (blue line), choroidal thickness (yellow line), and scleral thickness (dashed white double arrow). The height of the inward bulge of the retinal pigment epithelium (red double arrow) was measured above the tangent plane (red dashed line) at the bottom of the posterior pole. At points $2000 \mu \mathrm{m}$ superior and $2000 \mu \mathrm{m}$ inferior to the fovea, the choroidal (yellow line) and scleral (dashed white double arrow) thicknesses were measured. In the horizontal scan, choroidal and scleral thicknesses were also measured at $2000 \mu \mathrm{m}$ temporal and nasal to the fovea. White arrowheads indicate the outer scleral border.

Figure 2. Eyes with high myopia that show dome-shaped macular configuration within the posterior staphyloma. (Top left) Fundus photograph. (Top middle and right) Multi-averaged vertical (middle) and horizontal (right) scans through the fovea obtained by swept-source optical coherence tomography (OCT). (Top row) Both vertical and horizontal OCT scans show an inward convexity of the retinal pigment epithelium (RPE) within the staphyloma. The inward bulge is seen more clearly in the vertical scan. The top of the bulge (arrow) is located beneath the fovea. (2nd row) Both vertical and horizontal OCT scans show an inward convexity of the RPE. The top of the bulge (arrow) is located at the nasal side of the fovea. (3rd-5th rows) Vertical OCT scans show clearly an inward bulge of RPE within the posterior staphyloma. The top of the bulge (arrows) is located beneath the fovea. The horizontal scan shows an almost flat contour of the RPE.

Figure 3. An eye with high myopia that shows a band-shaped ridge within the posterior staphyloma. (Top left) Fundus photograph of the right eye of a 61-year-old woman (20/20 OD). Axial length was $28.60 \mathrm{~mm}$ and refractive error was -12.5 diopters. The dashed green rectangle outlines the area $\left(12 \times 8 \mathrm{~mm}^{2}\right)$ scanned by swept-source optical coherence tomography (OCT). (Top middle) Three-dimensional reconstructed image obtained by the swept-source OCT shows an inward convexity of the retina in the posterior pole. (Top right) Three-dimensional image reconstructed by segmentation of the retinal pigment epithelium (RPE) shows two outward concavities within the posterior staphyloma. A horizontal ridge is formed between these two outward concavities. (Bottom left) A vertical 12-mm line scan shows an inward bulge of the RPE beneath the fovea. The height of the RPE line above the tangent plane was $275 \mu \mathrm{m}$. The external 
surface of the sclera shows a rather smooth curvature (white arrowheads). The sclera shows marked thinning at the areas of the two concavities and seems to be relatively thick beneath the fovea. (Bottom right) A horizontal 12-mm line scan shows an almost flat contour of the RPE. The scleral thinning at the parafoveal area is less evident, compared with the areas of the two outward concavities within the staphyloma.

Figure 4. An eye with high myopia that shows a dome-shaped convexity within the posterior staphyloma. (Top left) Fundus photograph of the right eye of a 41-year-old woman (20/25 OD). Refractive error was -14.0 diopters and the axial length was 29.24 $\mathrm{mm}$. The dashed green rectangle outlines the area $\left(12 \times 8 \mathrm{~mm}^{2}\right)$ scanned by swept-source optical coherence tomography (OCT). Vertical and horizontal multi-averaged scans of $12 \mathrm{~mm}$ were obtained along the green arrows. (Top middle) Three-dimensional reconstructed image obtained by the swept-source optical coherence tomography shows an inward convexity of the retina in the posterior pole. (Top right) Three-dimensional image reconstructed by segmentation of the retinal pigment epithelium (RPE) shows the typical dome-shaped convexity within the staphyloma. (Bottom left) A vertical 12-mm line scan clearly shows an inward bulge of the RPE beneath the fovea. The height of the bulge of the RPE line above the tangent plane is $163 \mu \mathrm{m}$. The external surface of the sclera is seen clearly (white arrowheads) and the sclera shows uneven thinning in the posterior pole, being relatively thick beneath the fovea. (Bottom right) A horizontal 12-mm line scan also shows an inward convexity in the posterior pole. 
Table 1. Characteristics of Eyes with a Dome-Shaped Macula

Number of eyes/patients

Sex (male/female)

Age (years)

Axial length $(\mathrm{mm})$

Refractive error (diopters)

Visual acuity (logMAR)

Intraocular pressure $(\mathrm{mmHg})$

Height of inward bulge ${ }^{a}(\mu \mathrm{m})$

Foveal retinal thickness $(\mu \mathrm{m})$

Foveal choroidal thickness $(\mu \mathrm{m})$

Foveal scleral thickness $(\mu \mathrm{m})$

Type of posterior staphyloma

IX
I

II

III

1
$51 / 35$

$8 / 27$

$65.6 \pm 11.3$ (40 to 87$)$

$29.53 \pm 2.16$ (26.16 to 34.89$)$

$-13.69 \pm 5.86(-6.75$ to -31.0$)$

$0.36 \pm 0.39(-0.18$ to 1.40$)$

$14.8 \pm 2.7$ (9 to 22$)$

$152.3 \pm 58.8$ (56.0 to 294.0$)$

$190.9 \pm 84.9$ (0.0 to 522.5$)$

$34.5 \pm 29.0$ (10.0 to 117.5$)$

$518.6 \pm 97.6$ (316.0 to 711.0$)$
18

26

3

4

LogMAR, logarithm of minimal angle of resolution. ${ }^{2}$ The height of the inward bulge was measured from a presumed line tangent to the outer surface of retinal pigment epithelium at the bottom of the posterior staphyloma in the vertical section of optical coherence tomography. 
3D FEATURES OF DOME-SHAPED MACULA. Ellabban AA, et al.

Table 2. Choroidal and Scleral Thickness within the Macular Area in Eyes with a Dome-Shaped Macula

\begin{tabular}{|c|c|c|}
\hline & $\begin{array}{l}\text { Choroidal Thickness }(\mu \mathrm{m}) \\
\qquad\left(P \text { value }^{a}\right)\end{array}$ & $\begin{array}{l}\text { Scleral Thickness }(\mu \mathrm{m}) \\
\qquad\left(P \text { value }^{a}, P \text { value }^{b}\right)\end{array}$ \\
\hline Subfovea & $34.5 \pm 29.0$ & $518.6 \pm 97.6(\mathrm{NA},<.001)$ \\
\hline Parafovea at $2000 \mu \mathrm{m}$ superiorly & $49.8 \pm 37.8(.065)$ & $277.2 \pm 97.1(<.001,<.001)$ \\
\hline Parafovea at $2000 \mu \mathrm{m}$ inferiorly & $40.1 \pm 30.4(.869)$ & $284.9 \pm 91.1(<.001, .001)$ \\
\hline Parafovea at $2000 \mu \mathrm{m}$ temporally & $39.0 \pm 29.7(.940)$ & $306.0 \pm 97.3(<.001, .040)$ \\
\hline Parafovea at $2000 \mu \mathrm{m}$ nasally & $17.8 \pm 13.0(.035)$ & $360.3 \pm 93.5(<.001, \mathrm{NA})$ \\
\hline \multicolumn{3}{|c|}{${ }^{a} P$ values, compared with the values in the fovea; ${ }^{b} P$ values, compared with the values in the } \\
\hline 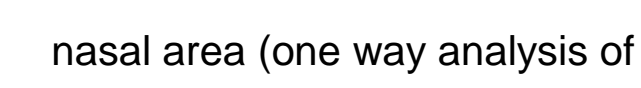 & ance with Tukey post ho & thaist \\
\hline
\end{tabular}


Table 3. Complications in the Eyes with a Dome-Shaped Macula

\begin{tabular}{lc}
\hline Choroidal neovascularization & $21(41.2 \%)$ \\
Serous retinal detachment & $3(5.9 \%)$ \\
Diffuse chorioretinal atrophy & $15(29.4 \%)$ \\
Patchy chorioretinal atrophy & $4(7.8 \%)$ \\
Lamellar macular hole & $3(5.9 \%)$ \\
Full-thickness macular hole & $1(1.9 \%)$ \\
Foveal schisis & $1(1.9 \%)$ \\
Extrafoveal retinal schisis & $9(17.6 \%)$ \\
\hline
\end{tabular}


Table 4. Characteristics of the Eyes with Dome-Shaped Macula with or without Choroidal Neovascularization

$\begin{array}{cc}\text { Eyes without } & \text { Eyes with } \\ \text { Choroidal } & \text { Choroidal }\end{array}$

Neovascularization Neovascularization $P$ value

Number of eyes (\%)

Sex (male/female)

Age (years)

Axial length $(\mathrm{mm})$

Refractive error (diopters)

Visual acuity (logMAR)

Foveal retinal thickness $(\mu \mathrm{m})$

Foveal choroidal thickness $(\mu \mathrm{m})$

Foveal scleral thickness $(\mu \mathrm{m})$

Height of the inward bulge ${ }^{c}(\mu \mathrm{m})$
$30(58.8 \%)$

$9 / 21$

$65.5 \pm 12.4$

$29.60 \pm 2.13$

$-13.61 \pm 6.26$

$0.24 \pm 0.32$

$190.1 \pm 62.0$

$38.5 \pm 32.8$

$518.0 \pm 109.7$

$167.3 \pm 59.5$
$21(41.2 \%)$

$2 / 19$

$.098^{a}$

$65.8 \pm 10.0$

$29.45 \pm 2.24$

$.816^{b}$

$-14.06 \pm 5.35$

$.806^{b}$

$0.53 \pm 0.42$

$.007^{b}$

$91.9 \pm 111.6$

$.943^{b}$

$28.8 \pm 21.8$

$.212^{b}$

$519.4 \pm 79.6$

$.958^{b}$

$131.0 \pm 51.7$

$.028^{b}$

${ }^{a}$ Fischer exact test. ${ }^{b}$ Unpaired t-test. ${ }^{c}$ Height of the inward bulge was measured from the presumed line tangent to the outer surface of retinal pigment epithelium at the bottom of the posterior staphyloma on vertical optical coherence tomography scans.

LogMAR, logarithm of minimal angle of resolution. 


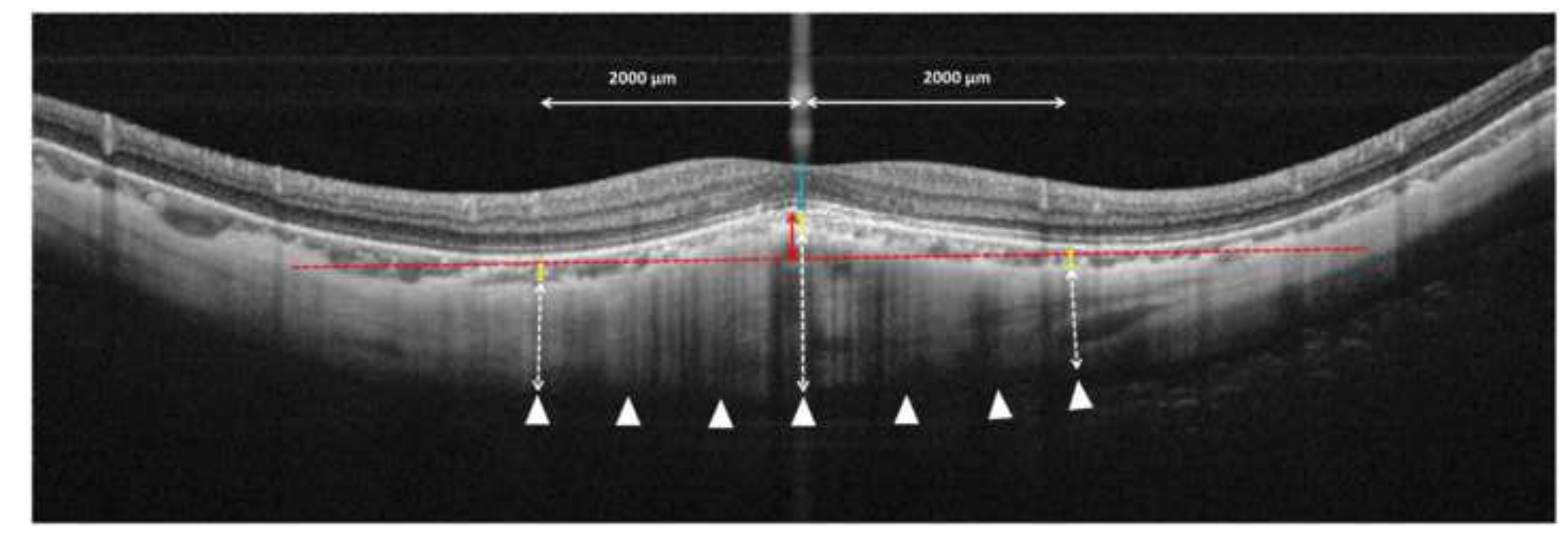

Click here to download high resolution 
Click here to download high resolution image

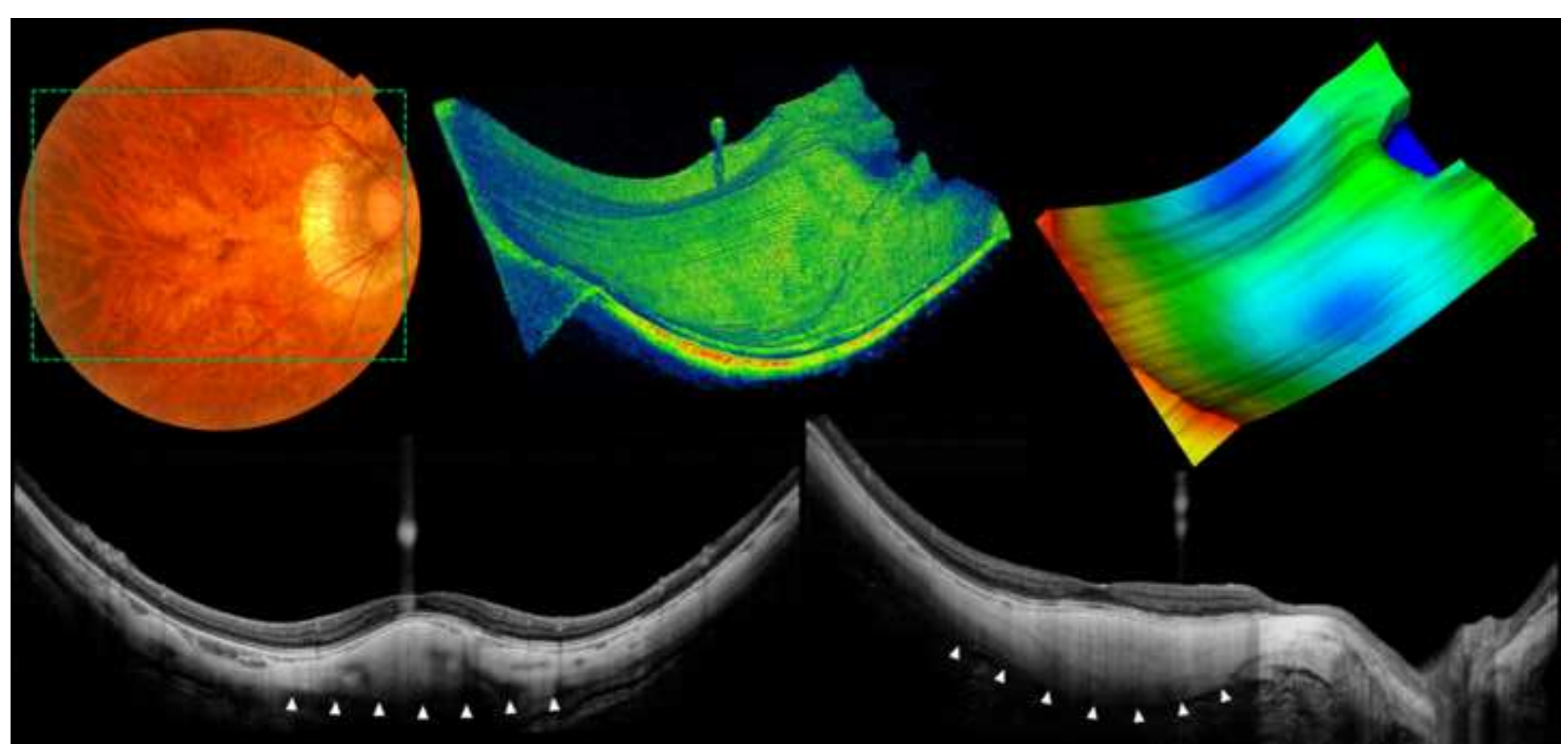

Click here to download high resolution image 
Click here to download high resolution image

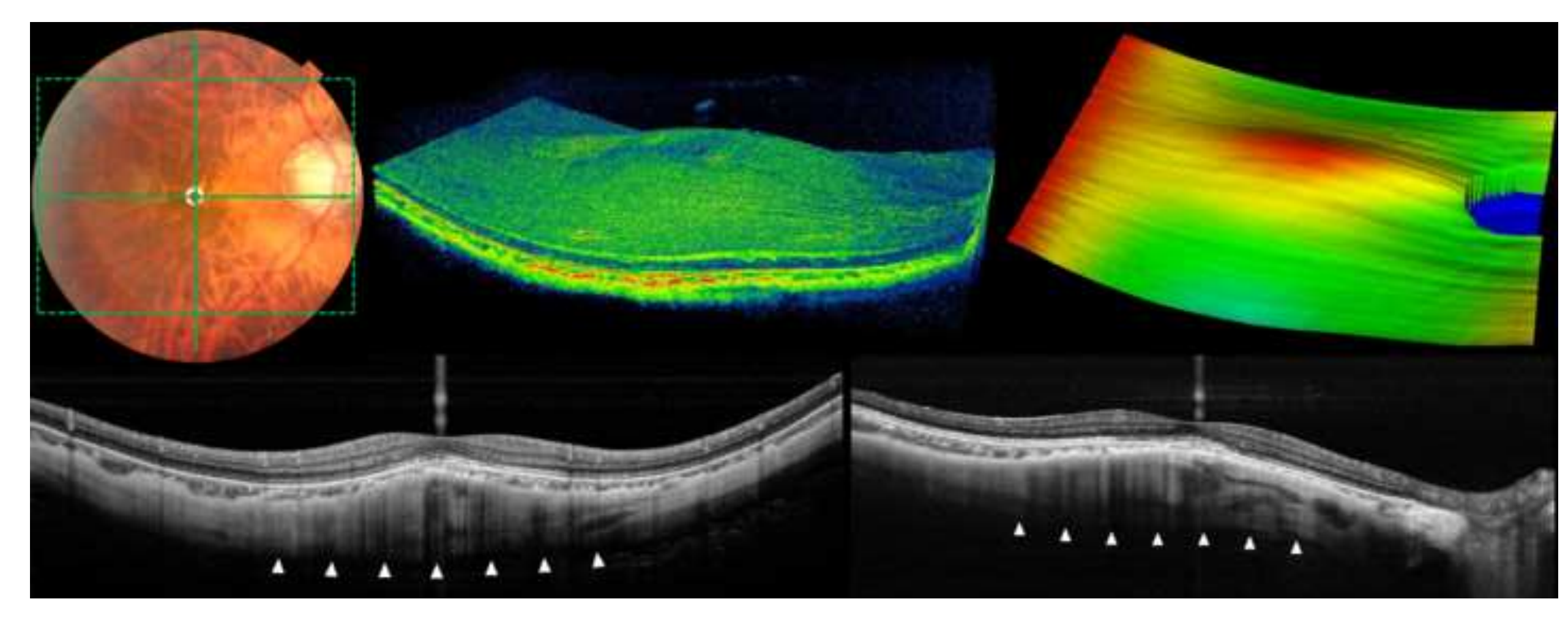


Figure 2
Click here to download high resolution image

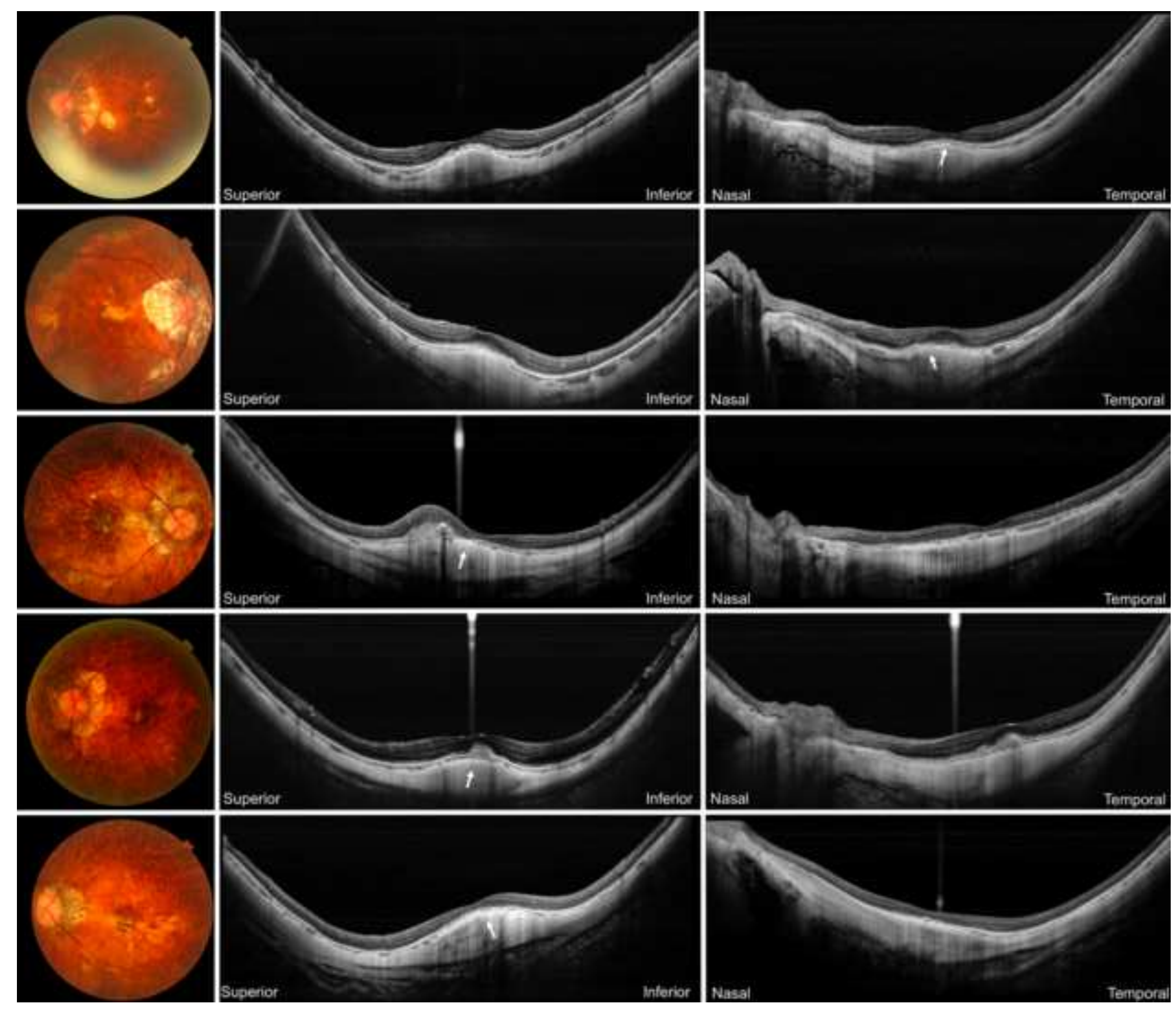




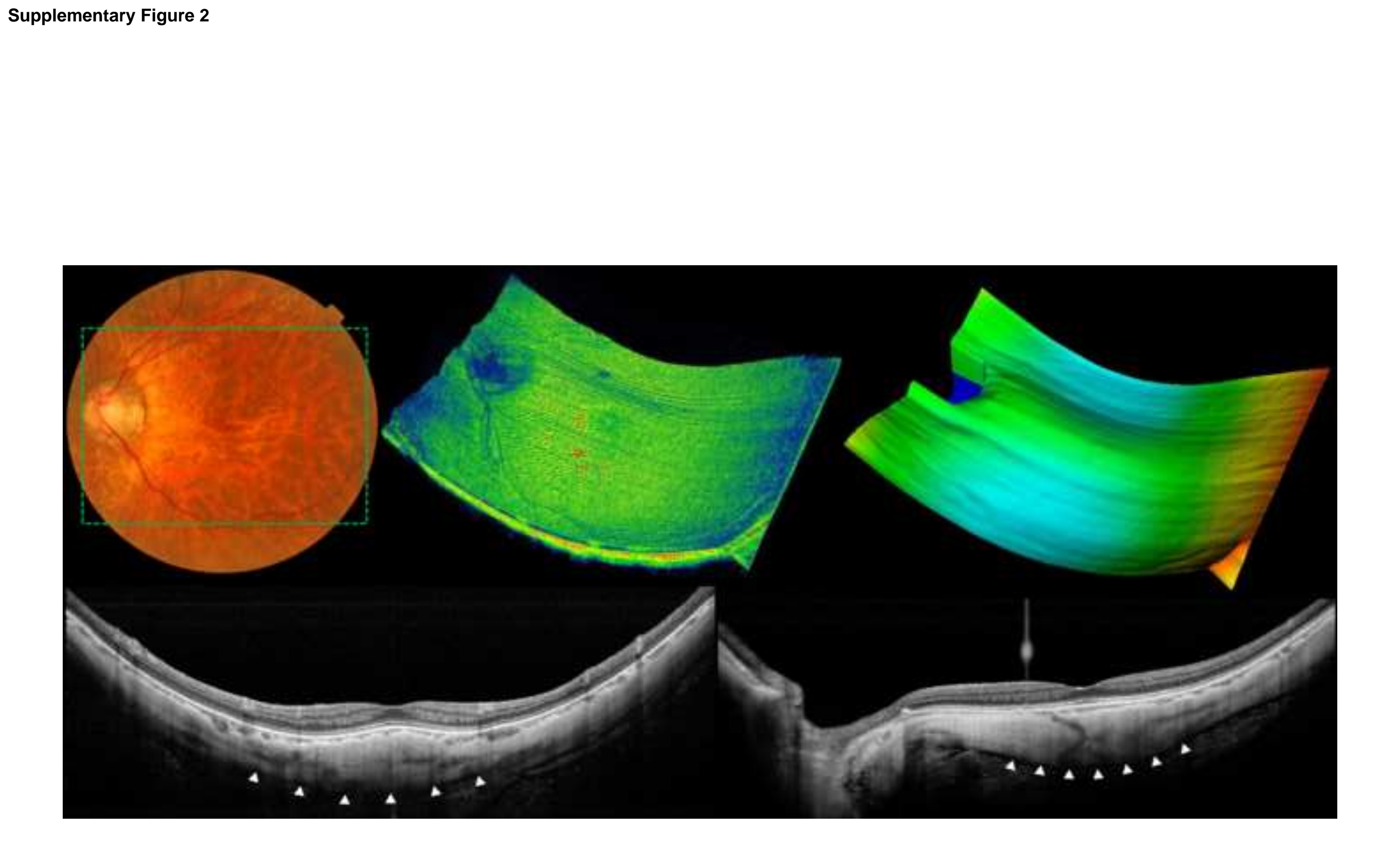

Supplementary Figure 2

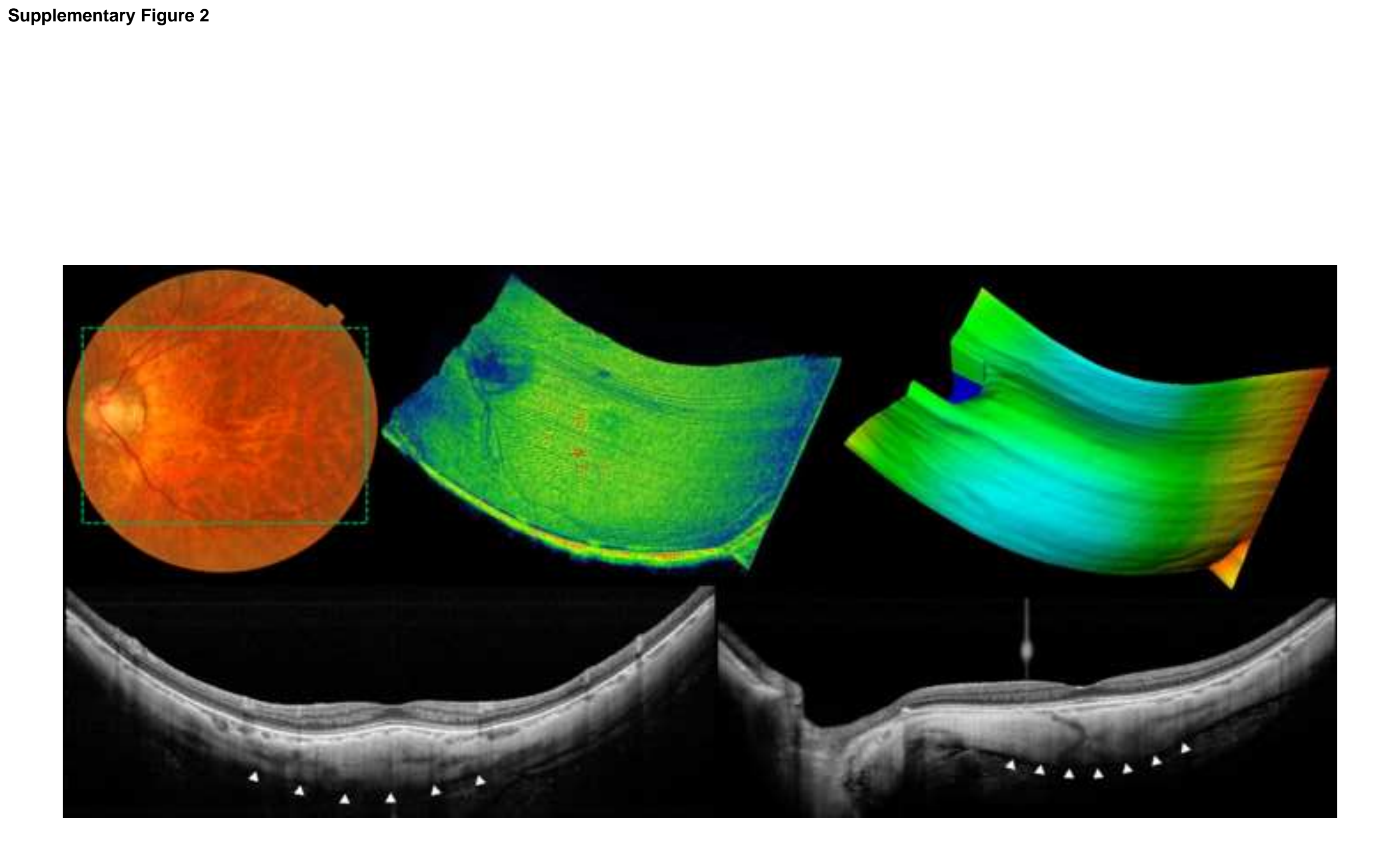

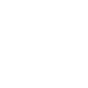

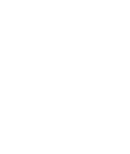

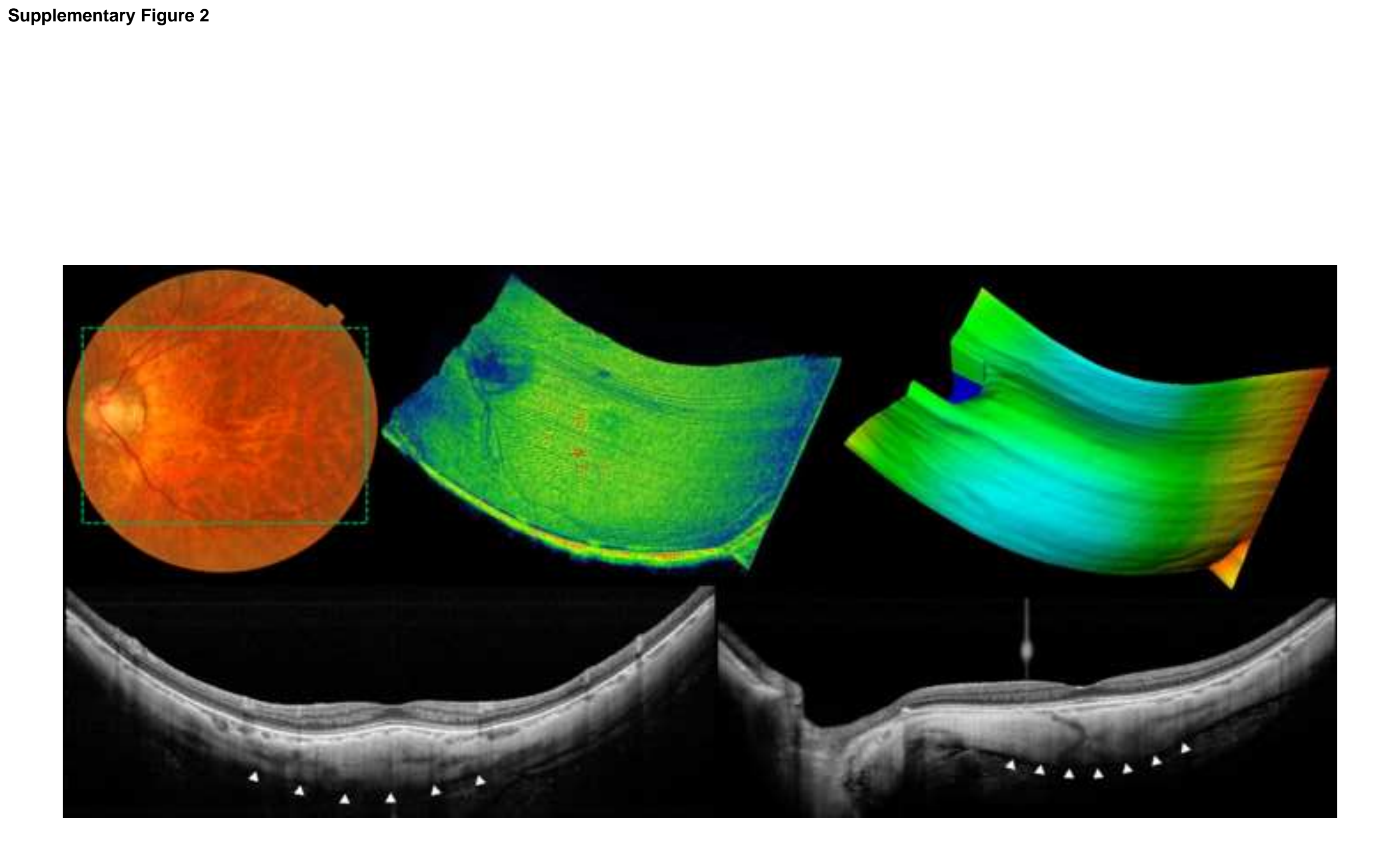




\section{Legend of Supplemental Material}

Supplemental figure 1. An eye with high myopia that shows a band-shaped ridge within the posterior staphyloma. (Top left) Fundus photograph of the right eye of a 42-year-old man (20/40 OD). Axial length was $29.19 \mathrm{~mm}$ and refractive error was -15.5 diopters. The dashed green rectangle outlines the area $\left(12 \times 8 \mathrm{~mm}^{2}\right)$ scanned by swept-source optical coherence tomography (OCT). (Top middle) Three-dimensional reconstructed image obtained by the swept-source OCT shows an inward convexity of the retina at the posterior pole. (Top right) Three-dimensional image of retinal pigment epithelium (RPE) shows two outward concavities within the posterior staphyloma. A horizontal ridge is formed between these two concavities. (Bottom left) A vertical 12-mm line scan shows an inward bulge of the RPE beneath the fovea. The height of elevation of the RPE line above the tangent plane is $277 \mu \mathrm{m}$. The external surface of the sclera shows a rather smooth curvature (white arrowheads). The sclera shows marked thinning in the areas of the two concavities and seems to be relatively thick beneath the fovea. (Bottom right) A horizontal 12-mm line scan shows that the contour of the RPE is almost flat. The scleral thinning at the parafoveal area is less evident, compared with the areas of the two outer concavities within the staphyloma.

Supplemental figure 2. An eye with high myopia that shows a band-shaped ridge within the posterior staphyloma. (Top left) Fundus photograph of the right eye of a 62-year-old woman (20/20 OD). Refractive error was -12.5 diopters. The dashed green rectangle outlines the area scanned by swept-source optical coherence tomography (OCT). (Top middle) Three-dimensional reconstructed image obtained by the swept-source OCT shows an inward convexity of the retina at the posterior pole. (Top right) Three-dimensional image of retinal pigment epithelium (RPE) shows two outward concavities within the staphyloma, with a horizontal ridge between these two concavities. (Bottom left) A vertical 12-mm line scan shows an inward bulge of the RPE beneath the fovea. The height of elevation of the RPE line above the tangent plane is $95 \mu \mathrm{m}$. The external surface of the sclera shows a rather smooth curvature (white arrowheads). The sclera shows marked thinning at the areas of the two concavities and seems to be relatively thick beneath the fovea. (Bottom right) A horizontal 12-mm line scan shows an almost flat contour of the RPE. The scleral thinning at the parafoveal area is less evident than within areas of the two outward concavities.

Supplemental Video 1. A three-dimensional movie of an eye with dome-shaped macular configuration (shown in Figure 3). Three-dimensional data sets covering an area of $12 \times 8$ 
3D FEATURES OF DOME-SHAPED MACULA. Ellabban AA, et al. Page 1

$\mathrm{mm}^{2}$ were obtained with swept-source optical coherence tomography using a raster scan protocol of 512 (horizontal) $\times 128$ (vertical) A-scans. The three-dimensional constructed image shows an inward convexity of the retina at the posterior pole. By segmentation of the retinal pigment epithelium line, a reconstructed image of the posterior pole clearly shows two outward concavities in the macular area with a horizontal ridge between these two concavities. 